\title{
Neurofibromatosis orbitotemporal gigante
}

\author{
Diego Gutiérrez*, Nicolás Urroz†, Marcelo Langleibł
}

\section{Resumen}

La neurofibromatosis orbitotemporal es una enfermedad progresiva que comienza en la infancia y puede generar importantes alteraciones morfológicas y funcionales. Presentamos el caso de un paciente adulto con diagnóstico de neurofibromatosis orbitotemporal gigante, en el cual se realizó un tratamiento mediante embolización preoperatoria y resección quirúrgica con el fin de disminuir el tamaño tumoral.

Palabras clave: Neurofibromatosis 1

Neurofibroma plexiforme

Embolización terapéutica

Cirugía plástica

Key words: $\quad$ Neurofibromatosis 1

Plexiform neurofibroma

Embolization Therapeutic

Plastic surgery

\section{Introducción}

Las neurofibromatosis son un grupo de enfermedades hereditarias que comprenden la neurofibromatosis tipo 1 (NF 1) o enfermedad de Von Recklinghausen, neurofibromatosis tipo 2 y la Schwannomatosis ${ }^{(1)}$.

La NF 1 es una enfermedad de herencia autosómica dominante con penetrancia completa y expresión variable, y una incidencia de 1 en 3.000 nacidos vivos aproximadamente ${ }^{(1)}$. Dentro de sus manifestaciones clínicas más características se encuentra la presencia de neurofi- bromas, que son tumores benignos de la envoltura de los nervios periféricos que se desarrollan a nivel de las partes blandas; alteraciones de la pigmentación de la piel, descritas como manchas café con leche; hamartomas pigmentados a nivel del iris, conocidos como nódulos de Lisch; glioma del nervio óptico; displasia de huesos largos y del ala mayor del esfenoides; tumores y mal formaciones a nivel del sistema nervioso central, entre otras $^{(2)}$

* Residente de la Cátedra de Cirugía Plástica, Reparadora y Estética, Hospital de Clínicas. Uruguay.

† Cirujano plástico. Asistente de la Cátedra de Cirugía Plástica, Reparadora y Estética, Hospital de Clínicas. Uruguay.

‡ Médico imagenólogo. Profesor Agregado del Departamento Clínico de Imagenología, Hospital de Clínicas. Uruguay.

Correspondencia:Diego Fernando Gutiérrez Melo. Correo electrónico: dfgutierrezm@gmail.com

Aspectos éticos: se obtuvo consentimiento informado y firmado del paciente para el registro fotográfico y para su inclusión en este trabajo.

Los autores declaran no tener conflicto de interés respecto al contenido de este trabajo

Recibido: $20 / 8 / 18$

Aceptado: 21/11/18 


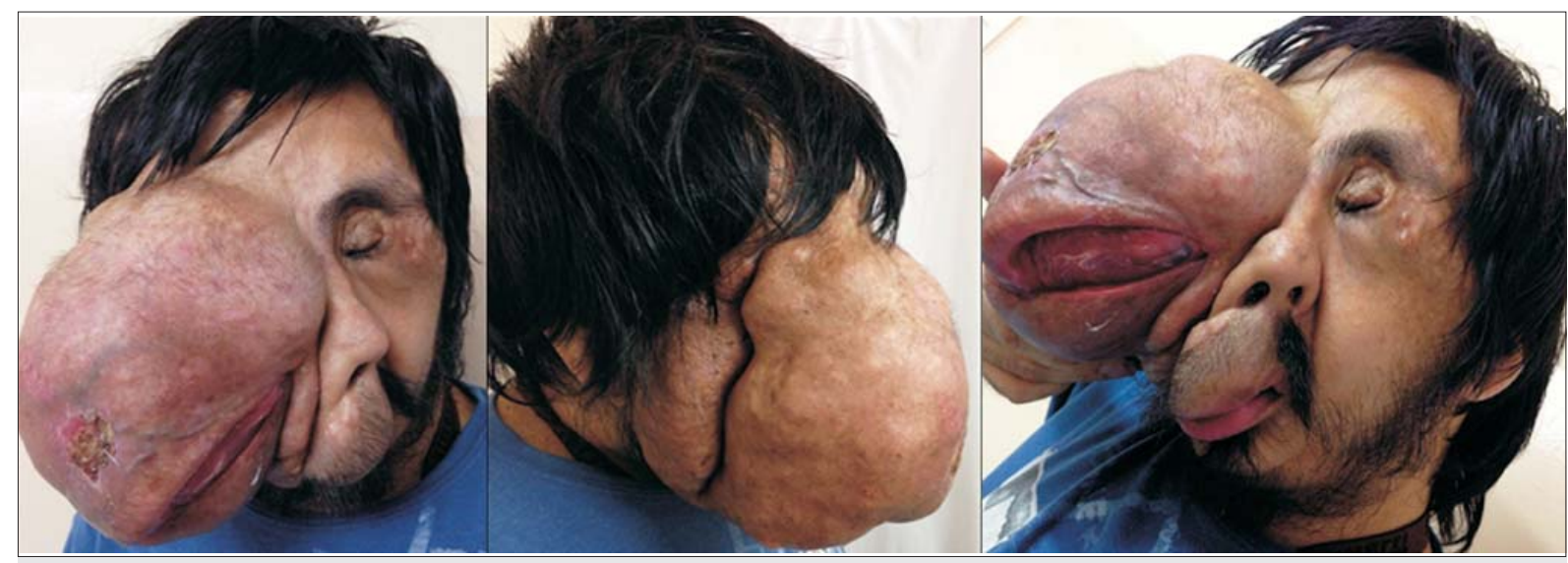

Figura 1. Fotos preoperatorias.

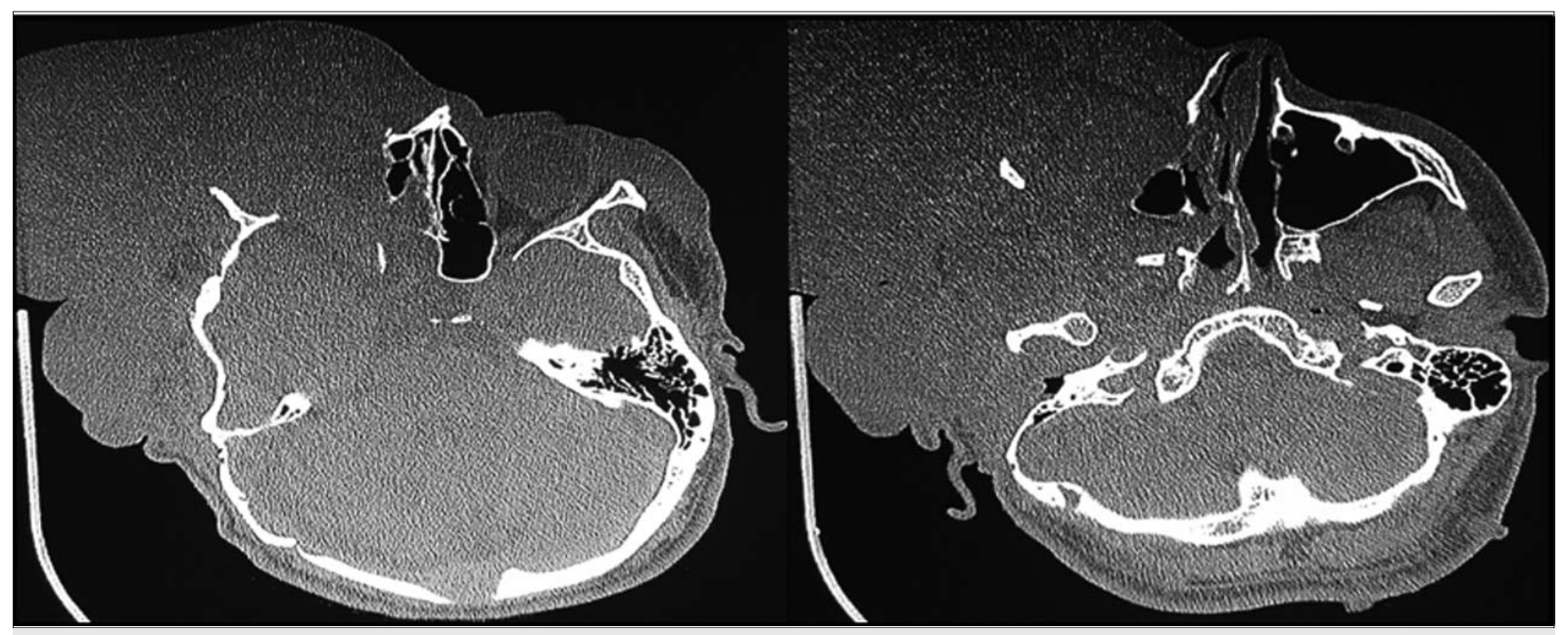

Figura 2. TAC de cráneo. Nótese la ausencia del ala mayor del esfenoides, la hipoplasia malar y maxilar, y el ensanchamiento orbitario.

Existe un subgrupo de pacientes, dentro de la NF 1, en los que predomina la neurofibromatosis plexiforme progresiva a nivel orbitotemporal, asociada a alteraciones orbitarias, pudiendo comprometer la visión del ojo $\operatorname{afectado}^{(1)}$.

Presentamos un caso clínico de un paciente con una neurofibromatosis orbitotemporal gigante, tratado en Hospital de Clínicas de Montevideo, Uruguay.

\section{Caso clínico}

Paciente de sexo masculino, de 27 años, con diagnóstico de NF 1 desde la infancia. Consultó en la cátedra de Cirugía Plástica en el Hospital Universitario presentando una gran tumoración en hemicara derecha, con crecimiento progresivo desde la niñez. Refiere que durante la pubertad el neurofibroma aumentó rápidamente de tamaño, lo cual le generó una gran repercusión psicológica y aislamiento social. A los 16 años se sometió a una cirugía en la que se realizó una resección parcial del tumor. Luego de la misma, no concurre más a controles ni asistencia médica hasta la consulta actual, 11 años más tarde.

Se trata de un paciente con un nivel intelectual menor al esperado para su edad, que abandonó la escuela en la infancia y no trabaja. Vive en el fondo de la casa de un familiar, que es la única persona con que socializa habitualmente.

Al examen, se destaca una neurofibromatosis plexiforme orbitotemporal a derecha de gran tamaño que desplaza las estructuras de la línea media, con proptosis, blefaroptosis y ceguera del ojo derecho (figura 1). A la palpación no presenta pulsaciones ni frémito. Además, presenta nódulos de Lisch, manchas color café con leche en el cuerpo y neurofibromas subcutáneos pequeños en el tronco. En la tomografía computada se evidencia agrandamiento y distopia de la órbita, con ausencia del ala mayor del esfenoides, hipoplasia malar y desplaza- 


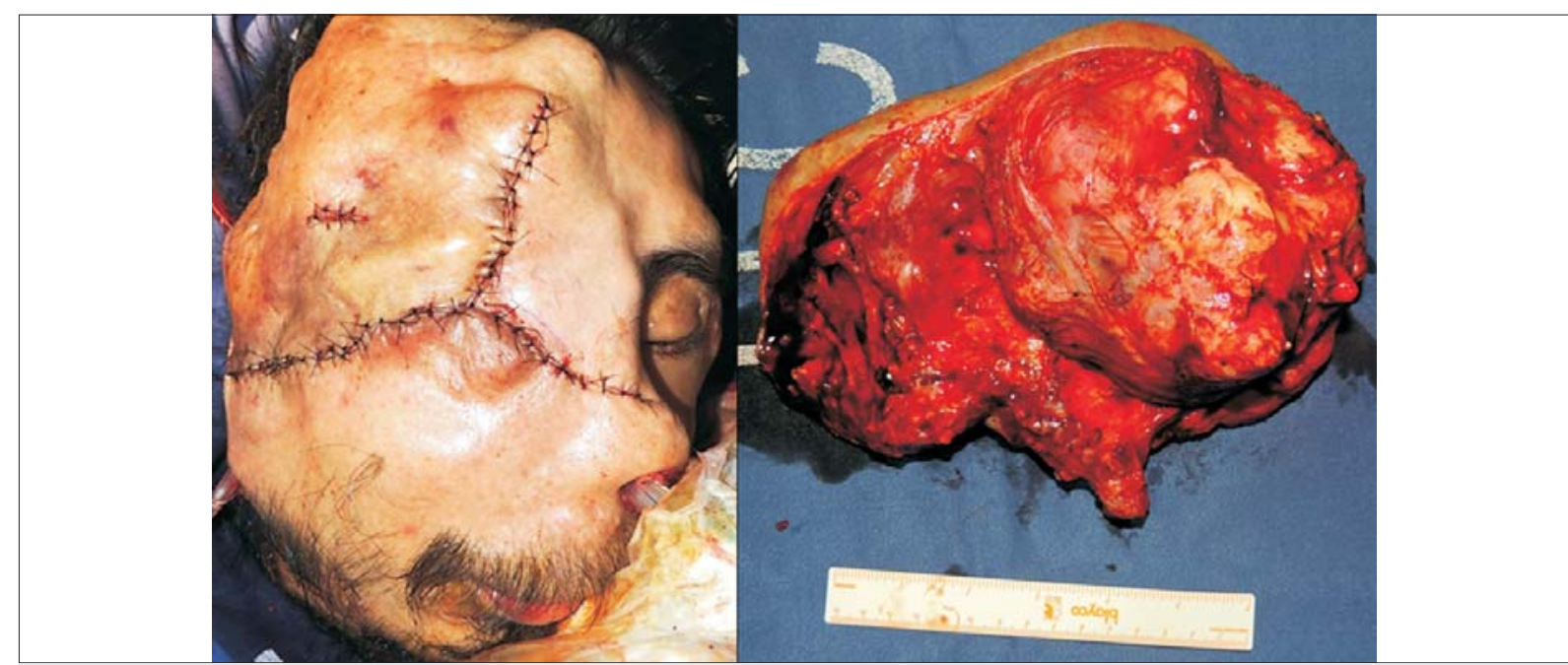

Figura 3. Fotos posoperatorias.

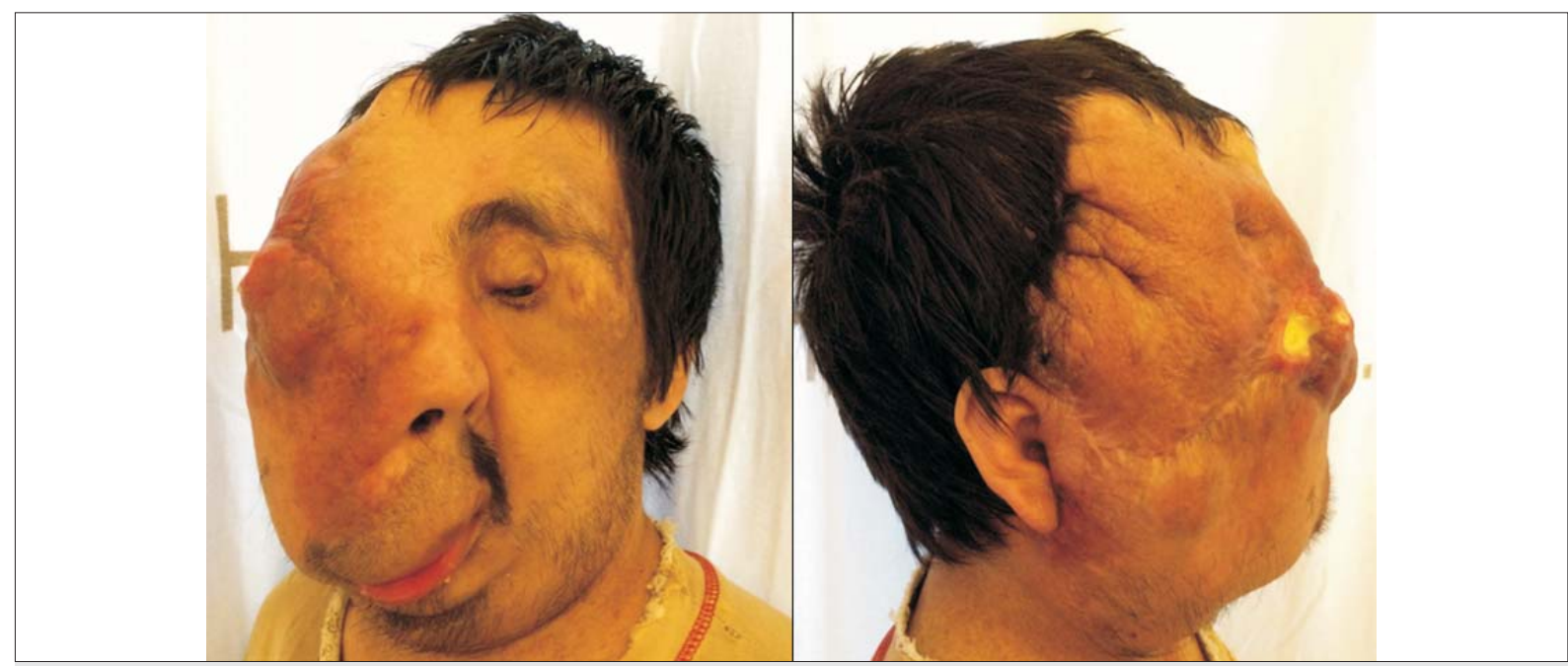

Figura 4. Fotos posoperatorias.

miento de las fosas nasales y el tabique nasal hacia la izquierda. A nivel de partes blandas, presenta una masa con densidad heterogénea que compromete el sector intra y extraconal de la órbita desplazando el globo ocular en sentido anterior y lateral, y se extiende ampliamente por las partes blandas extracraneanas fronto-parieto-temporales (figura 2).

En vistas al tratamiento quirúrgico, considerando el riesgo de complicaciones hemorrágicas por el tamaño y la topografía del neurofibroma, solicitamos una arteriografía con embolización preoperatoria a los efectos de disminuir la vascularización y el riesgo de sangrado; 48 horas antes de la cirugía se realizó la arteriografía diagnóstica selectiva de las arterias carótida interna y externa ipsilaterales a la lesión, y luego la cateterización superselectiva con microcatéter de las arterias que irriga- ban la lesión, embolizando con micropartículas de alcohol polivinílico los vasos distales e intratumorales.

La cirugía se realizó bajo anestesia general e intubación orotraqueal. La resección fue siguiendo un plano tangente al macizo facial, ligando vasos oftálmicos y resecando el globo ocular derecho en conjunto con la pieza. La cobertura se realizó mediante la confección de tres colgajos dermograsos (figura 3 ).

\section{Resultados}

Se logró una disminución significativa de la masa del tumor, disminuyendo el peso y el dolor. Logramos un buen control de la hemostasis durante la cirugía y no tuvimos complicaciones hemorrágicas intraoperatorias ni posoperatorias. 
En el posoperatorio inmediato se constató sufrimiento isquémico a nivel distal de los colgajos de piel, resultando en un área cruenta pequeña que cicatrizó por segunda intención (figura 4).

En la evolución, el paciente asiste a los controles de forma esporádica pero no acepta más intervenciones quirúrgicas. El estudio anatomopatológico de la lesión confirmó el diagnóstico de neurofibroma y ausencia de malignidad.

\section{Discusión}

La neurofibromatosis orbitotemporal comienza a desarrollarse en la vida intrauterina y habitualmente crece durante la infancia, pero en algunos casos puede mantenerse quiescente hasta la pubertad, donde aumenta de tamaño y continúa creciendo en la vida adulta ${ }^{(3)}$. Se estima que afecta entre $1 \%$ a $10 \%$ de los pacientes con NF $1^{(4)}$.

En estos pacientes la neurofibromatosis infiltra las partes blandas de la órbita, párpados y una extensión variable de la región temporal, generalmente de forma unilateral, pudiendo asociar una extensión intracraneana. Puede disminuir la capacidad visual del ojo afectado y en los casos más severos produce ceguera. A nivel óseo, presentan una ausencia parcial o completa del ala mayor del esfenoides, produciendo un defecto de la pared posterolateral de la órbita y un ensanchamiento de la hendidura esfenoidal. Como resultado, el lóbulo temporal puede prolapsarse hacia la órbita exacerbando el exoftalmos y transmitiendo las pulsaciones al globo ocular. Los pacientes pueden presentar también un ensanchamiento de la órbita que adquiere forma oval, con una hipoplasia malar y una depresión del piso de la órbita ${ }^{(5,6)}$. Generalmente, asocian otros signos sistémicos característicos de la NF $1^{(1)}$.

El tratamiento de los neurofibromas es quirúrgico, si bien existen actualmente quimioterapias en fase de ensayo clínico ${ }^{(7-9)}$. La táctica quirúrgica depende en gran medida de la extensión y severidad de la enfermedad, pero en general se trata de cirugías complejas y con resultados subóptimos, siendo un tratamiento desafiante y frustrante para el cirujano y el paciente.

Jackson y colaboradores clasifican la NF orbitotemporal en tres grupos según su severidad: 1. Compromete solo partes blandas de la órbita, conservando la capacidad visual. 2. Afecta las partes blandas y el continente óseo de la órbita que está agrandado, conserva la capacidad visual. 3. Compromiso óseo y de partes blandas de la órbita con ceguera o ausencia de globo ocular ${ }^{(5)}$. El caso clínico presentado corresponde al grado 3 de esta clasificación, dado que presenta una infiltración severa de todas las estructuras orbitarias, ceguera del ojo derecho y un marcado agrandamiento del continente óseo. Para el tratamiento de pacientes con este grado de severidad,
Jackson y colaboradores proponen la exenteración del contenido orbitario, reconstrucción de las paredes óseas con injerto óseo y cobertura de la cavidad con colgajos de piel, para la adaptación luego de una prótesis externa de párpados y ojo ${ }^{(5)}$. En nuestro paciente, dado el gran tamaño del neurofibroma, el objetivo de esta intervención fue disminuir la masa tumoral.

Los neurofibromas son tumores muy vascularizados que infiltran de forma difusa los tejidos y frecuentemente presentan malformaciones vasculares como senos venosos, dilataciones aneurismáticas y fístulas arteriovenosas $^{(10,11)}$, determinando un riesgo elevado de hemorragia durante la cirugía, e incluso se han descrito hemorragias espontáneas ${ }^{(12)}$. Littlewood y Stilwell fueron los primeros en proponer la embolización previa a la resección quirúrgica de neurofibromas plexiformes ${ }^{(11)}$. Desde entonces, el desarrollo de las técnicas intravasculares, así como el descubrimiento de nuevos agentes embolizantes, han convertido a la embolización preoperatoria en una herramienta valiosa en el manejo de tumores muy vascularizados $^{(13)}$.

En nuestro paciente, dado el tamaño del neurofibroma y su topografía, decidimos realizar una embolización previo a la cirugía. Si bien no hubo complicaciones hemorrágicas durante la cirugía ni en el posoperatorio, pensamos que la isquemia en el sector distal de los colgajos de cobertura podría deberse a la embolización preoperatoria.

En general, se recomienda el tratamiento y seguimiento de estos tumores desde la infancia ${ }^{(6)}$ dado el fuerte impacto que genera en la autoestima y en el desarrollo social del individuo y su entorno familiar. En el caso de nuestro paciente, lo llevó al aislamiento social y al abandono del tratamiento cuando era más joven. Proponen también que la cirugía temprana podría evitar el agrandamiento orbitario, la ptosis palpebral y el compromiso del globo ocular ${ }^{(5)}$.

Por otra parte, la transformación maligna de estos tumores es una complicación poco frecuente, está descrito que se presenta en hasta $4 \%$ a $5 \%$ de los neurofibromas plexiformes ${ }^{(1)}$. En nuestro paciente, el estudio anatomopatológico de la pieza descartó la presencia de degeneración maligna.

\section{Conclusiones}

La neurofibromatosis orbitotemporal es una patología compleja y su tratamiento debe adaptarse a cada paciente según la severidad de la enfermedad y las deformidades que presente. El tratamiento quirúrgico de estos tumores puede llevar a complicaciones hemorrágicas. De acuerdo con la evidencia de otros autores, pensamos que la embolización preoperatoria, en el caso clínico que presentamos, permitió un mejor control de la he- 
mostasis durante la cirugía y disminuyó el riesgo de hemorragia posoperatoria.

\section{Abstract}

Orbitotemporal neurofibromatosis is a progressive disease that begins in childhood and may cause important morphological and functional alterations. We present the case of an adult patient with a giant orbitotemporal neurofibromatosis, which was treated by preoperative embolization and a surgical resection to reduce the size of the tumor size.

\section{Resumo}

A neurofibromatose órbito-temporal é uma enfermidade progressiva que começa na infância e pode gerar importantes alterações morfológicas e funcionais. Apresentamos o caso de um paciente adulto com diagnóstico de neurofibromatose órbito-temporal gigante, que foi submetido a tratamento com embolização pré-operatória e ressecção cirúrgica para diminuir o tamanho tumoral.

\section{Bibliografía}

1. Kapur S, Bentz ML. Pediatric Tumors. En: Rodriguez ED, Losee JE, ed. Craneofacial, Head and Neck surgery. Pediatric Plastic Surgery. 3 ed. Plastic Surgery; vol 3 Neligan Peter C, serie ed, Londres. Elsevier Saunders, 2013:877-92

2. Ferner RE, Gutmann DH. Neurofibromatosis type 1 (NF1): diagnosis and management. En: Said G, Krarup C, ed. Peripheral Nerve Disorders. Handbook of Clinical Neurology, 3 serie, Vol 115. Amsterdam. Elsevier BV, 2013: 939-55.

3. Lee V, Ragge NK, Collin RO. The surgical management of childhood orbito-temporal neurofibromatosis. Br J Plast Surg 2003; 56(4): 380-87.

4. Havlik RJ, Boaz J. Cranio-orbital-temporal neurofbromatosis: Are we treating the whole problem? J Craniofac Surg 1998;9:529-35.
5. Jackson IT, Carbonnel A, Potparic Z, Shaw K. Orbitotemporal neurofibromatosis: classification and treatment. Plast Reconstr Surg 1993; 92(1): 1-11.

6. Latham K, Buchanan EP, Suver D, Gruss JS. Neurofibromatosis of the head and neck: Classification and surgical management. Plast Reconstr Surg 2015; 135(3): 845-55.

7. Hirbe AC,Gutmann DH. Neurofibromatosis type 1: A multidisciplinary approach to care. Lancet Neurol 2014; 13(8): 834-43.

8. Katowitz WR, Katowitz JA. Orbitofacial Neurofibromatosis 1: Current Medical and Surgical Management. En: Guthoff RF, Katowitz JA, eds. Essentials in ophthalmology. Oculoplastics and Orbit, Berlin. Springer, 2010:79-92

9. Li J, Lin M, Shao Ch, Ge S, Fan X. Blepharoplasty techniques in the management of orbito-temporal neurofibromatosis. J Plast Reconstr Aesthet Surg 2014; 67(11) 1496-501.

10. Oderich GS, Sullivan TM, Bower TC, Gloviczki P, Miller DV, Babovic-Vuksanovic D, Macedo TA, Stanson A. Vascular abnormalities in patients with neurofibromatosis syndrome type I: clinical spectrum, management, and results. J Vasc Surg 2007; 46: 475-84.

11. Littlewood AHM, Stilwell JH. The vascular features of plexiform neurofibroma with some observations on the importance of pre-operative angiography and the value of pre-operative intra-arterial embolisation. $\mathrm{Br} \quad \mathrm{J}$ Plast Surg 1983;36:501-06.

12. Tomei KL, Gupta V, Prestigiacomo ChJ, Gandhi ChD. Spontaneous hemorrhage of a facial neurofibroma: endovascular embolization before surgical intervention. J Craniofac Surg 2013;24(5):e514-17.

13. Nair S, Gobin YP, Leng LZ, Marcus JD, Bilsky M, Laufer I, Patsalides A. Preoperative embolization of hypervascular thoracic, lumbar, and sacral spinal column tumors: Technique and outcomes from a single center. Interv Neuroradiol 2013;19: 377-385. 